ORIGINAL ARTICLE

\title{
No GIST-type c-kit gain of function mutations in neuroblastic tumours
}

\author{
M Korja, J Finne, T T Salmi, H Haapasalo, M Tanner, J Isola
}

J Clin Pathol 2005;58:762-765. doi: 10.1136/jcp.2004.024331

See end of article for authors' affiliations ......................

Correspondence to: $\operatorname{Dr} M$ Korja, Department of Medical Biochemistry and Molecular Biology, University of Turku Kiinamyllynkatu 10, FI20520 Turku, Finland; miikka.korja@utu.fi

Accepted for publication 14 December 2004

\begin{abstract}
Aims: Neuroblastic tumours (NTs) have been shown to respond to imatinib treatment in vivo and in vitro, possibly via inactivating the c-kit receptor. The purpose of this study was to identify gastrointestinal stromal tumour (GIST)-type c-kit gene associated mutations in exons $9,11,13$, and 17 in NTs to recognise a subset of tumours that would probably respond to imatinib treatment.

Methods: Expression of the c-kit protein was detected immunohistochemically in a total of 37 archival paraffin wax embedded NTs using polyclonal rabbit antihuman c-kit antibody. After immunohistochemistry, c-kit gene associated chromosomal mutations in all cases of NT were detected with denaturing high performance liquid chromatography (HPLC).

Results: Denaturing HLPC analysis did not reveal GIST-type mutations in four immunohistochemically detected c-kit positive or in $33 \mathrm{c}$-kit negative NTs.

Conclusions: c-kit receptor expression and GIST-type c-kit gene mutations are rare events in NTs. Oncogenic activation of c-kit in NTs presumably differs from that of GISTs, which may influence their responsiveness to imatinib treatment. Whether c-kit has an essential role in the pathogenesis of NTs remains to be investigated.
\end{abstract}

T he protooncogene c-kit encodes a growth factor receptor that possesses autophosphorylation activity. ${ }^{1}$ Autophosphorylation is induced by the binding of haemopoietic stem cell factor, a pleiotropic cytokine, which has been identified as a ligand for the c-kit receptor tyrosine kinase. ${ }^{2}$ c-kit plays a central role in gastrointestinal stromal tumours (GISTs), which are the most common mesenchymal neoplasms in the human digestive tract. Almost all GISTs distinctively express the c-kit receptor, and up to $92 \%$ of GISTs have gain of function mutations, ${ }^{3}$ which primarily $(87.5 \%)$ localise to exons 9 and 11 of the c-kit gene. ${ }^{4}$ In addition, gain of function mutations in exons 13 and 17 of the c-kit gene have been reported. ${ }^{35}$ Gain of function mutations of the c-kit gene activate tyrosine phosphorylation without stem cell factor binding, which results in autonomous growth of cells in vitro and in vivo. ${ }^{6}$ Imatinib is a competitive inhibitor of several structurally related receptor tyrosine kinases, which include Bcr-Abl, Abl related gene, platelet derived growth factor receptor, and most interestingly, c-kit. Imatinib treatment of a patient with unresectable, metastatic, and chemotherapy resistant GISTs has resulted in dramatic clinical, radiological, and pathological improvement. ${ }^{7}$ The mutational status of c-kit has been regarded as an independent predictor of clinical response to imatinib.

\section{"The development of new therapeutic agents and strate- gies effectively inhibiting tumour cell growth in patients with unresectable and metastatic stage 4 neuroblastic tumours continues to be a therapeutic challenge"}

Neuroblastic tumours (NTs) (neuroblastoma, ganglioneuroblastoma, and ganglioneuroma) evolve from the primordial neural crest and are the most common extracranial solid tumours in children. The development of new therapeutic agents and strategies effectively inhibiting tumour cell growth in patients with unresectable and metastatic stage 4 NTs continues to be a therapeutic challenge. Immortal NT cell lines, ${ }^{8-11}$ and primary tumours, ${ }^{8} 1012$ express the c-kit receptor. Stem cell factor is believed to have a trophic role in neuroblastoma cells through an autocrine growth loop, which leads to the activation of the c-kit receptor. ${ }^{8}$ Interestingly, imatinib can inhibit neuroblastoma cell proliferation, possibly via inhibition of c-kit in vitro ${ }^{10}{ }^{11}$ and in vivo. ${ }^{11}$ Thus, it might be possible to broaden the field of clinical applications of imatinib to other c-kit positive solid malignancies, such as NTs. To date, however, only exon 11 GIST-type c-kit gene mutations have been examined in a set of 40 NTs. $^{12}$ The purpose of our present study was to characterise GIST-type c-kit gene associated mutations in exons 9, 11, 13, and 17. If such mutations exist in NTs, these tumours would probably respond to imatinib treatment.

\section{METHODS}

\section{Clinical samples}

In total, 37 archival, paraffin wax embedded NTs from the years 1967-2001 were obtained from Turku University Hospital and Tampere University Hospital, Finland. The local ethics committee approved our study. Among these 37 cases, 34 tumour samples were obtained from the primary site and 35 samples before treatment. One primary tumour was intracranial. ${ }^{13}$ For histological typing and grading, the international neuroblastoma pathology classification (International Classification) was applied. ${ }^{14}$ The intracranial tumour was not included in the classification. These 34 classified tumours comprised 31 NTs (14 undifferentiated, eight poorly differentiated, and nine differentiating), two ganglioneuroblastomas (one nodular, one intermixed), and one ganglioneuroma. Of these 34 cases, nine had favourable and 25 had unfavourable histology.

\footnotetext{
Abbreviations: GIST, gastrointestinal stromal tumour; HPLC, high performance liquid chromatography; NT, neuroblastic tumour; PCR, polymerase chain reaction
} 
Table 1 Primers and annealing temperatures for amplification of c-kit exons

\begin{tabular}{lllll}
\hline Exon & Forward primers & Reverse primers & Fragment size & Annealing temperature ( $\mathrm{C}$ ) \\
\hline 9 & GTATGCCACATCCCAAGTGT & CATGACTGATATGGTAGACA & 334 & 56 \\
11 & CCAGAGTGCTCATGACTG & GGAAGCCACTGGAGTTCCT & 274 & 56 \\
13 & GACATCAGTTGCCAGTTGT & TGTITGATAACCTGACAGAC & 214 & 56 \\
17 & GCAACACTATAGTATTAAAAAG & CCTITGCAGGACTGTCAAGCA & 371 & TD $68^{*}$ \\
\hline \multirow{2}{*}{ *Touchdown polymerase chain reaction with annealing temperature decreasing by $0.5^{\circ} \mathrm{C}$ every cycle to a "touchdown" annealing temperature of $60^{\circ} \mathrm{C}}$.
\end{tabular}

\section{c-kit immunohistochemistry and detection of MYCN amplification status}

Expression of the c-kit protein was detected immunohistochemically in paraffin wax embedded sections using polyclonal rabbit antihuman c-kit antibody (CD117; 1/250 dilution; DakoCytomation, Glostrup, Denmark) after microwave antigen retrieval. Briefly, the microwave antigen retrieval step consisted of three seven minute long heating periods in Tris/EDTA buffer ( $\mathrm{pH} 9.0)$ in a microwave oven $(850 \mathrm{~W})$. Immunostaining was performed in an automated immunostainer (TechMate 500 Plus; DakoCytomation) using the avidin-biotin complex method with diaminobenzidine as the chromogen. A GIST tumour served as a positive control. Mast cells served as internal positive controls and as a reference for positive immunolabelling. Immunostaining in more than $30 \%$ of the cells was considered to be a positive reaction. ${ }^{10}$ A cutoff value of $10 \%$ has also been used in one report, ${ }^{12}$ but this does not hamper the comparison of the results, because normally more than $50 \%$ of the tumour cells were labelled in positive cases. Immunolabelling for c-kit was evaluated in a simple blind trial fashion by three independent observers. MYCN amplification status in NTs was assessed with chromogenic in situ hybridisation ( $\mathrm{M}$ Korja et al, unpublished, 2004). A tumour was considered to be MYCN amplified when a mean of six or more nuclear signals were seen for each 100 nuclei, or when tumour nuclei showed large clustered signals. No adjustment for potential hyperploidy was made.

\section{Denaturing HPLC analysis of c-kit mutations}

Exons 9, 11, 13, and 17 of the c-kit gene were evaluated for the presence of mutations. Genomic DNA was extracted and purified from paraffin wax embedded tumour specimens using a QIAamp mini kit according to the manufacturer's recommended protocol for paraffin wax embedded tissue samples (Qiagen, Hilden, Germany). Genomic DNA (50 ng) was amplified in a polymerase chain reaction (PCR) containing $0.6 \mu \mathrm{l}$ Platinium PCR buffer (Invitrogen, Carlsbad, California, USA), $\quad 1.4-2.4 \mathrm{mM} \quad \mathrm{MgCl}_{2}, \quad 160 \mu \mathrm{M}$ dNTPs (Clontech, Palo Alto, California, USA), $0.3 \mu \mathrm{M}$ forward and reverse primers, and DNA polymerases-AmpliTaq Gold (1.25 U; Applied Biosystems, Branchburg, New Jersey, USA) and Platinium Taq (1.25 U; Invitrogen) -in a $50 \mu \mathrm{l}$ volume. Negative and positive (GIST tumour) controls were included in every batch of amplifications. Table 1 lists the forward and reverse oligonucleotide primers used to amplify c-kit exons 9, 11, 13, and 17.

Denaturing high performance liquid chromatography (HPLC) analysis was carried out using Agilent Technologies' series 1100 HPLC system (Agilent Technologies, Palo Alto, California, USA). The PCR product $(5-10 \mu \mathrm{l})$ was injected into the Zorbax Eclipse double stranded DNA HPLC column $(75 \times 2.1 \mathrm{~mm}$; Agilent Technologies) and eluted at a flow rate of $0.4 \mathrm{ml} /$ minute within a linear acetonitrile gradient, consisting of a mixture of buffer A (100mM triethylammonium acetate and $0.1 \mathrm{mM}$ EDTA; Varian Inc, Walnut Creek, California, USA) and buffer
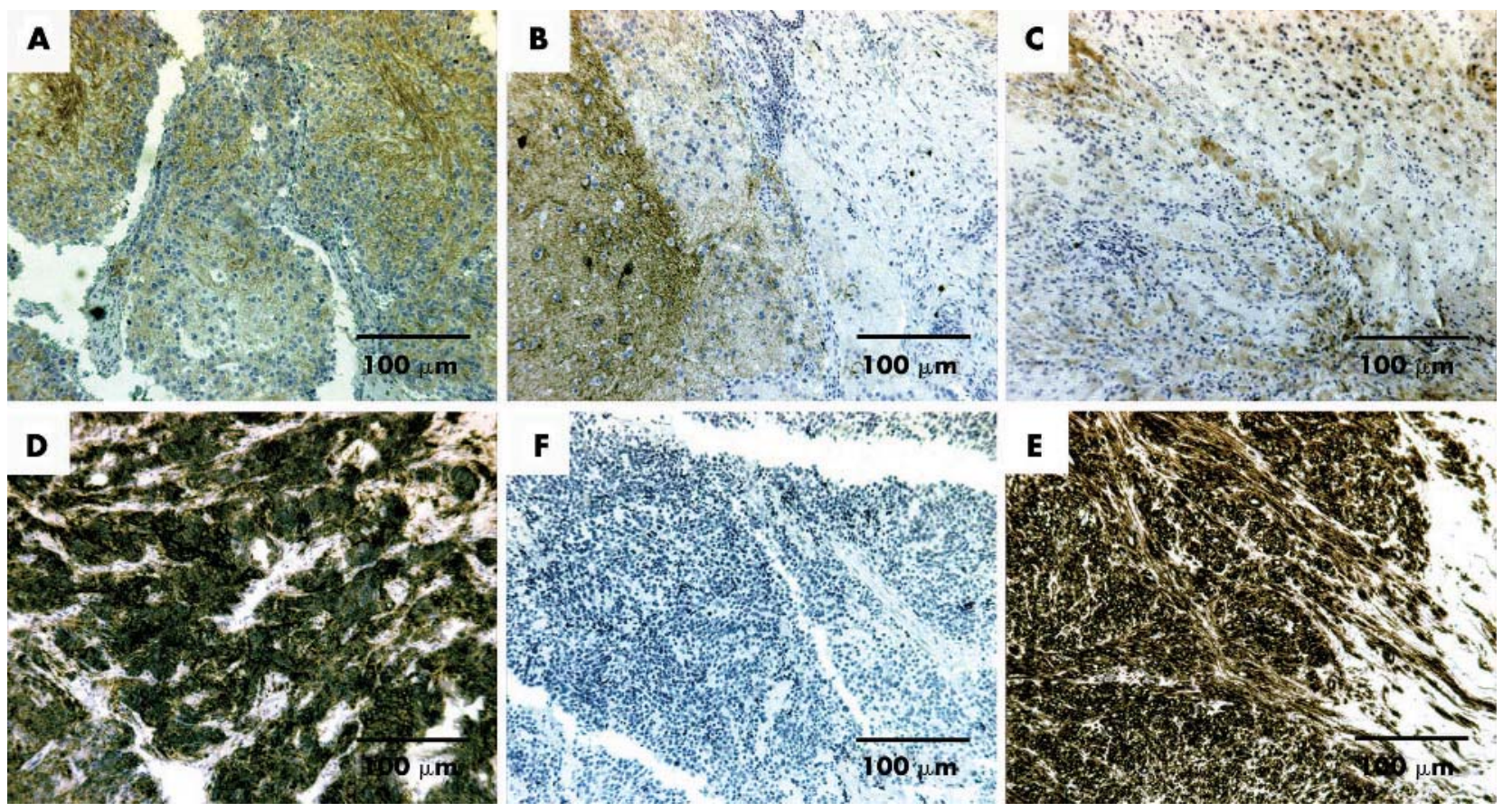

Figure 1 Immunostaining for c-kit in (A-D) human neuroblastic tumours (NTs) and (E) gastrointestinal stromal tumour. (F) A negative NT control obtained by omission of the primary antibody. 
B (100mM triethylammonium acetate, 0.1mM EDTA, and $25 \%$ acetonitrile; Varian Inc). The DYS271 standard, which consists of a 209 base pair fragment of heterozygous double stranded DNA, with an A to G mismatch at position 168, was used as a control. The elution temperatures for each amplicon were obtained from the denaturing HPLC Melt Program (http://insertion.stanford.edu/melt.html), and then optimised by studying alterations in the elution profiles of the samples within a temperature range of $3{ }^{\circ} \mathrm{C}$ under and above the suggested melting temperature. The temperature that best separated homoduplexes was used for denaturing HPLC analysis.

Samples with abnormal-like elution profiles in denaturing HPLC were subjected to automated sequencing. The PCR products were first purified using Montage DNA purification columns (Millipore, Bedford, Massachusetts, USA). Direct sequencing of PCR products was performed using BigDye 3 termination chemistry (Applied Biosystems, Foster City, California, USA) and an ABI 3100 genetic analyser (Applied Biosystems), according to the instructions provided by the manufacturer.

\section{RESULTS}

Tumour phenotyping with the anti-c-kit antibody detected expression of the c-kit receptor in four of the 37 tumours tested (fig 1). More than $30 \%$ of the tumour cells were labelled in all positive cases. In general, the labelling intensity of positive NTs was much weaker than the GIST tumours (fig 1). The low prevalence of c-kit expressing NTs is in accordance with previous results. ${ }^{9}{ }^{10}$ Furthermore, only one of eight MYCN amplified NTs was c-kit positive. The number of positive c-kit immunopositive samples in our study was too small to define a statistical relation between c-kit expression and common prognostic factors of NTs. Nevertheless, table 2 summarises the age, prognostic group (International Classification), and MYCN amplification status of all four ckit positive tumours.

Because the mutational status has been shown to have a central role in GISTs, we analysed all our NT samples for the presence of mutations in exons 9, 11, 13, and 17 of the c-kit gene. No c-kit gene mutations were found in the 37 NT samples. An abnormal-like elution pattern suspicious for a mutation was found in one tumour in exon 11. However, bidirectional sequence analysis revealed the normal wild-type sequence, with no signs of a mutation. This tumour was immunohistochemically c-kit negative and had no MYCN amplification.

\section{DISCUSSION}

GISTs present a variety of gain of function mutations across the c-kit gene. These mutations result in a constitutively activated c-kit receptor, leading to malignant growth potential. ${ }^{6}$ In GISTs, the clinical response to imatinib treatment is related to the gain of function mutational status of the c-kit gene. ${ }^{4}$ The current series of NTs is the first that has been examined for GIST-type c-kit gene mutations in

Table 2 Summary of the features of c-kit positive neuroblastic tumours

\begin{tabular}{|c|c|c|c|}
\hline IC & $\begin{array}{l}\text { Patient's age } \\
\text { (days) }\end{array}$ & $\begin{array}{l}\text { Prognostic } \\
\text { group }\end{array}$ & $\begin{array}{l}\text { MYCN } \\
\text { amplification }\end{array}$ \\
\hline NB (differentiating)* & 4939 & - & - \\
\hline NB (differentiating) & 4879 & Unfavourable & - \\
\hline NB (differentiating) & 549 & Favourable & - \\
\hline NB (undifferentiated) & 909 & Unfavourable & + \\
\hline
\end{tabular}

*Intracranial tumour. ${ }^{13}$

IC, International Classification; NB, neuroblastic tumour. exons 9, 11, 13, and 17. Based on our results, GIST-type c-kit mutations are rare events in NTs.

We detected c-kit expression in $10.8 \%$ of NTs. In two previous studies the proportions of c-kit expressing NTs varied between 10 of 75 NTs (13\%) and 50 of 155 tumours $(32.3 \%) .{ }^{10}{ }^{12}$ NTs are intratumorally heterogeneous, and immunohistochemical staining results of various markers can vary between different groups. In addition, different sample materials (for example, histology, stage, location, pretreatments, etc) and methodological differences have some influence on the results. Some low level c-kit expressing NTs probably escape detection by immunohistochemistry. It has been previously reported that immunohistochemically negative NTs were either negative for c-kit by reverse transcription PCR, or contained low amounts of c-kit mRNA. ${ }^{12}$ However, because c-kit expression does not seem to be associated with c-kit mutations in NTs, the immunohistochemical staining result does not influence the c-kit mutation analysis.

\section{"Expression of the c-kit receptor without gain of function mutations of the c-kit gene is probably a pathogenetically less important phenotypic alteration"}

Recently, c-kit expression was preferentially found in the most aggressive subset of NTs-those harbouring genomic amplification of the MYCN oncogene. ${ }^{10}$ In contrast, another study showed that c-kit expression in NTs was not associated with MYCN amplification, but with a favourable prognosis. ${ }^{12}$ Because of this discrepancy, we also analysed the MYCN status of NTs. Because only one case of c-kit positive and MYCN amplified neuroblastoma was found in our series, no statistical significance can be demonstrated. All four c-kit positive tumours were neuroblastomas according to the International Classification. ${ }^{14}$

We found no GIST-type c-kit mutations in NTs. Despite the rarity of c-kit mutations, some NTs express the "unmutated" c-kit receptor, which may contribute to tumour proliferation or survival in vivo and in vitro. If the c-kit receptor plays a role in the pathogenesis of NTs, imatinib treatment might be a new therapeutic approach for this malignancy. It has been reported that imatinib inhibits the growth of c-kit positive neuroblastoma cells in vitro, and causes an inhibition of stem cell factor induced phosphorylation of the c-kit receptor in neuroblastoma cell lines. ${ }^{11}$ In addition, mice treated orally with imatinib every 12 hours for 14 days in a gastrocnemius neuroblastoma xenograft model had significantly smaller tumours than control mice. ${ }^{11}$ However, a previously published study demonstrates imatinib driven growth inhibition also in a c-kit negative neuroblastoma cell line. ${ }^{10}$ Accordingly, imatinib may suppress the growth of neuroblastoma through c-kit independent pathways-for example, through the platelet derived growth factor receptor and/or vascular endothelial growth factor. ${ }^{11}$ Thus, expression of the c-kit receptor without gain of function mutations of the c-kit gene

Take home messages

- c-kit receptor expression and gastrointestinal stromal tumour (GIST)-type c-kit gene mutations are found in only a small proportion of neuroblastic tumours (NTs)

- The oncogenic activation of c-kit in NTs presumably differs from that of GISTs, which may influence their responsiveness to imatinib treatment

- Whether c-kit has an essential role in the pathogenesis of NTs remains to be investigated 
is probably a pathogenetically less important phenotypic alteration.

In conclusion, the expression of the c-kit receptor in only a low proportion of NTs and the paucity of GIST-type c-kit mutations suggest that the therapeutic activity of imatinib through c-kit kinase suppression would benefit at most only a small subset of patients suffering from NTs. The molecular subclassification of NTs on the basis of c-kit expression does not appear to help identify tumours bearing c-kit mutations. c-kit mutations may not contribute to the pathogenesis of NTs. Whether c-kit has any role to play in the growth of NTs or the transition of neural crest cells to NTs remains to be investigated.

\section{ACKNOWLEDGEMENTS}

We thank Mrs S Toivola, Ms R Randen, and Mrs L Lempiäinen for skilful technical assistance.

\section{Authors' affiliations}

M Korja, J Finne, Department of Medical Biochemistry and Molecular Biology, University of Turku, Kiinamyllynkatu 10, Fl-20520 Turku,

Finland

T T Salmi, Department of Paediatrics, Turku University Central Hospital, Turku, Fl-20520 Finland

H Haapasalo, Department of Pathology, Tampere University Hospital, Fl-33521 Tampere, Finland

J Isola, Laboratory of Cancer Biology, Institute of Medical Technology, University of Tampere and Tampere University Hospital,

Fl-33014 Tampere, Finland

M Tanner, Department of Oncology, Tampere University Hospital, $\mathrm{FI}-33520$

\section{REFERENCES}

1 Yarden Y, Kuang W-J, Yang-Feng T, et al. Human proto-oncogene c-kit: a new cell surface receptor tyrosine kinase for an unidentified ligand. EMBO J 1987; 11:3341-51.

2 Williams DE, Eisenman J, Baird A, et al. Identification of a ligand for the c-kit proto-oncogene. Cell 1990;63:167-74.

3 Rubin BP, Singer S, Tsao C, et al. KIT activation is a ubiquitous feature of gastrointestinal stromal tumors. Cancer Res 2001;61:8118-21.

4 Heinrich MC, Corless CL, Demetri GD, et al. Kinase mutations and imatinib response in patients with metastatic gastrointestinal stromal tumor. J Clin Oncol 2003;23:4342-49.

5 Lux ML, Rubin BP, Biase TL, et al. KIT extracellular and kinase domain mutations in gastrointestinal stromal tumors. Am J Pathol 2000;156:791-95.

6 Hirota S, Isozaki K, Moriyama Y, et al. Gain-of-function mutations of c-kit in human gastrointestinal stromal tumors. Science 1998;279:577-80.

7 Joensuu H, Roberts PJ, Sarlomo-Rikala M, et al. Effect of the tyrosine kinase inhibitor STI571 in a patient with a metastatic gastrointestinal stromal tumor. N Engl J Med 2001;344:1052-6.

8 Cohen PS, Chan JP, Lipkunskaya M, et al. Expression of stem cell factor and ckit in human neuroblastoma. Blood 1994;10:3465-72.

9 Beck D, Gross C, Brognara B, et al. Expression of stem cell factor and its receptor by human neuroblastoma cells and tumors. Blood 1995;8:3132-8.

10 Vitali R, Cesi V, Nicotra MR, et al. c-Kit is preferentially expressed in MYCNamplified neuroblastoma and its effect on cell proliferation is inhibited in vitro by STI-571. Int J Cancer 2003;106:147-52.

11 Beppu K, Jaboine J, Merchant M, et al. Effect of imatinib mesylate on neuroblastoma tumorigenesis and vascular endothelial growth factor expression. J Natl Cancer Inst 2004;96:46-55

12 Krams M, Parwaresch R, Sipos B, et al. Expression of the c-kit receptor characterizes a subset of neuroblastomas with favorable prognosis. Oncogene 2004;23:588-95.

13 Ahdevaara P, Kalimo H, Torma T, et al. Differentiating intracerebral neuroblastoma: report of a case and review of the literature. Cancer 1977;40:784-8.

14 Shimada H, Ambros IM, DehnerLP, et al. Terminology and morphologic criteria of neuroblastomas: recommendations by the international neuroblastoma pathology committee. Cancer 1999;86:349-63. 